\title{
Vertrauenswürdige Kommunikation durch dezentrale Technologien: Editorial
}

\author{
Andrea Herrmann ${ }^{1}$ \\ (c) Springer-Verlag GmbH Deutschland, ein Teil von Springer Nature 2020
}

Mit zunehmender Digitalisierung wird immer mehr elektronisch kommuniziert, beruflich wie privat. Vertrauliche, persönliche, geschäftskritische, sicherheitskritische oder geheime Daten gehen über Datenleitungen von einem Computer zu einem anderen und werden oft zentral und längerfristig an einem Ort gesammelt. Firmen betreiben Customer Relationship Management, zahlreiche Unternehmen der New Economy bieten ihre Dienstleistungen kostenlos an und verdienen Geld nur mit den gesammelten Nutzerdaten. Das bringt zahlreiche, wachsende Risiken nicht nur für den Datenschutz unserer persönlichen Daten mit sich.

Ist dieser Trend unumkehrbar? Dieses Themenheft diskutiert zahlreiche Lösungsansätze, wie diese Entwicklung verlangsamt oder sogar umgekehrt werden kann:

- In seinem Artikel „Datenschutz und Internet - Es ist kompliziert!“ zeigt Peter Schaar anhand eines historischen Rückblicks, dass das Internet ursprünglich nicht für die Übermittlung persönlicher Daten und für den Datenschutz erfunden wurde. Gesetze wie die Datenschutz-Grundverordnung und die E-Privacy-Richtlinie versuchen diesen Mangel zu reparieren. Wichtig ist es, auch im Netz die zentralen ethischen und rechtlichen Prinzipien zu verteidigen!

- Im Internet tobt ein Krieg der Giganten um die Monopolisierung. Das Kartellrecht soll die Interessen der Menschen wahren. Vorschläge für rechtliche Maßnahmen für Daten- und Verbraucherschutz macht der Artikel „Ungezähmte Internetgiganten - GWB-Digitalisierungsgesetz: Trippelschritte mit geringer Wirkung - Für eine digitale Grundversorgung im 21. Jahrhundert!“ von UnternehmensGrün, Initiative „Konzernmacht beschränken“, Oxfam Deutschland und digitalcourage.

\footnotetext{
Andrea Herrmann

andrea.herrmann@fh-dortmund.de
}

1
- Der Artikel „Die Rückkehr des Dezentralen - Wie sich Internetaktive gegen Regulierung wehren" von Joachim Selzer zeigt im historischen Rückblick, dass Dezentralität zu den ursprünglichen Prinzipien des Internets gehört. Mit Hilfe von Onion Routing kann man auch heutzutage noch anonym surfen. Das Darknet hat auch Schattenseiten, denn es bietet Gut und Böse Datenschutz. Aber Anonymität ist ein wichtiges Bedürfnis.

- Durch die Initiative „Holen wir uns das Web zurück“ (,The web is ours“) (Kai Bösefeldt) werden die Nutzerinnen und Nutzer, Programmiererinnen und Programmierer dazu aufgerufen, nur Systeme zu verwenden, bei denen das Privacy-by-Design-Prinzip umgesetzt ist, und gemeinsam solche zu entwickeln. Er diskutiert den Unterschied zwischen zentralisierten, föderalen und verteilten Architekturen und was sie für den Datenschutz bedeuten.

- Eine technische Lösung besteht in dezentralen Technologien, die keine zentrale Datensammlung anhäufen. In meinem Artikel „Vertrauenswürdige Kommunikation: Ergebnisse einer Umfrage" stelle ich drei Initiativen, einige Peer-to-Peer-Netzwerke und die Ergebnisse einer Umfrage innerhalb der Gesellschaft für Informatik vor. Für zahlreiche bekannte Datenkraken gibt es datenschutzfreundliche alternative Software und Communities, die nur noch nicht genügend bekannt sind.

Ich wünsche eine interessante Lektüre, und vielleicht wollen Sie sich ja zukünftig auf die eine oder andere Weise für die vertrauenswürdige Kommunikation engagieren. Die genannten Initiativen suchen noch Programmierer/innen, Aktive und Nutzer/innen.

Dr. Andrea Herrmann 


\section{Anhang}

\section{Zum Titelbild}

Computing potentially visible geometry set (PVS) for any camera offset within a supported region for large dynamic meshes is a complex problem that scales with the amount of geometry. Computing PVS for practical camera offset ranges in real-time on current hardware is computationally demanding, as every triangle needs to be compared to many other triangles, possibly merging into larger occluder regions, on a per-frame basis. The picture shows a birdseye view on a full 360 degree potentially visible set (PVS) for a camera offset region computed using The Camera Offset Space from four reference views (in the corners) in an complex outdoor scene. Geometry labeled as invisible is shown in magenta color, the red dot shows camera position in the scene. Each reference view contains a corner inset depicting its corresponding PVS-starting from top right, clockwise: North, East, South and West. The final PVS is constructed by joining these 4 sets. Novel views rendered with the PVS for view points around the reference location are complete and hole free. This method opens new possibilities for frame-rate up-sampling and is particularly beneficial to streaming rendering systems and VR (Fig. 1).

Jozef Hladky, Max-Planck-Institut für Informatik, Saarbrücken

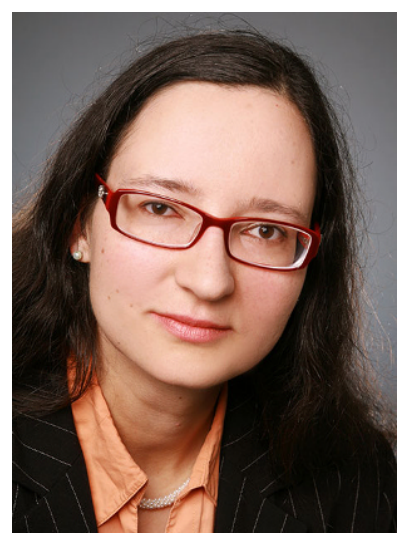

Andrea Herrmann

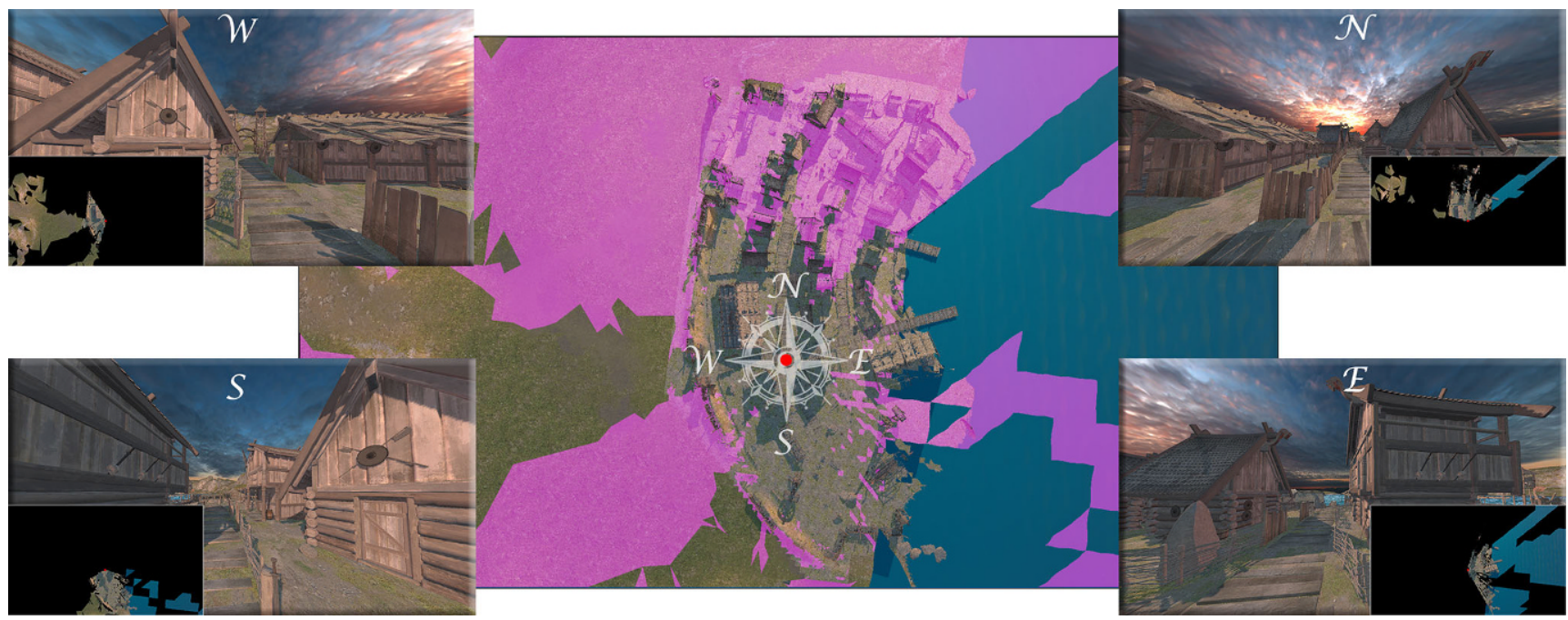

Fig. 1 Birds-eye view on a full 360 degree potentially visible set (PVS) computed using the Camera Offset Space 